\title{
The effect of residual lens aberrations on the determination of column positions around partial dislocations in GaAs
}

\author{
X. Xu ${ }^{*, *}$, S.P. Beckman ${ }^{*, * *}$, P. Specht ${ }^{*}$, D.C. Chrzan ${ }^{* * * *}$, E.R. Weber ${ }^{* * * *}$, C. Kisielowski ${ }^{* *}$ \\ *Dept. of Materials Science and Engineering, University of California, Berkeley, CA 94720 \\ **National Center for Electron Microscopy, Lawrence Berkeley national Laboratory, Berkeley, CA \\ 94720 \\ ***Material Science Division, Lawrence Berkeley National Laboratory, Berkeley, CA 94720
}

In recent years, it became possible to extend the resolution of field emission microscopes into the sub Angstrom region by reconstruction of the electron exit wave from a focus series of lattice images [1-4]. This improved resolution makes it desirable to revisit imaging and analyses of dislocation core structures with truly atomic resolution. A clarification of the atom arrangements in dislocation cores is a long standing problem [5].

A focus series of lattice images from edge-on dislocations in GaAs:Be were recorded using the Philips CM300 FEG/UT microscope at National Center for Electron Microscopy. Figure 1 is a phase image of a reconstructed electron exit wave from a wedge shaped GaAs sample that shows two partial dislocations with the adjacent stacking fault. Both partial dislocations are of $30^{\circ}$ character as determined by a Burgers circuit. The inserted extra half plane ends between narrowly spaced (111) planes revealing a glide-set configuration. Figure 2 shows a second example with a stacking fault intersecting the sample surface and a $30^{\circ} \mathrm{Ga}$ terminated partial dislocation. We explore a quantitative characterization of $30^{\circ}$ partial dislocations by matching experiments with $a b$ initio electronic structure total energy calculations employing ultrasoft pseudo potentials [6, 7]. Combining the $a b$ initio results with simulation software enables calculations with a similar number of atoms as recorded in the experiment [8]. Agreement between theory and experiment is quantified by comparing column positions extracted from experiment and simulation. It is of special interest to estimate the effect of residual lens aberrations on our ability to match column positions. Hardware corrections of lens aberrations by an analyses of Thon rings allow for control of residual distortions in the reconstructed exit wave image to about $50 \mathrm{pm}$. Such measurements become unreliable if the residual distortions are smaller. In this case, a comparison with theory is utilized that reveals discrepancies of $\sim 20 \mathrm{pm}$ between calculated and measured column positions at non-periodic lattice sites indicating that the residual distortions are around such lower values. A further reduction of image distortions by aberration correction through software is as shown in Figure 3 and will be discussed $[9,10]$.

\section{REFERENCES}

[1] C. Kisielowski et al., Ultramicroscopy 89 (2001) 243.

[2] A. Thust et al., Ultramicroscopy 64 (1996) 211.

[3] W.M.J. Coene et al., Ultramicroscopy 64 (1996) 109.

[4] M. A. O'Keefe et al., Ultramicroscopy 89 (2001) 215.

[5] V. Alexander et al., Philosophical Magazine Letters 79 (1999) 9.

[6] G. Kress, J. Fürthmuller, Physical Review B 54, (1996) 11169.

[7] G. Kress, J. Fürthmuller, Computational Material Science 6, (1996) 15.

[8] S.P. Beckman et al., J. Phys. 14 (2003) 12673. 
[9] A. Thust et al., Ultramicroscopy 64 (1996) 249.

[10] The project was sponsored by the Director, Office of Science, Office of Basic Energy Sciences, of the U.S. Department of Energy under Contract No. DE-AC03-76SF00098.

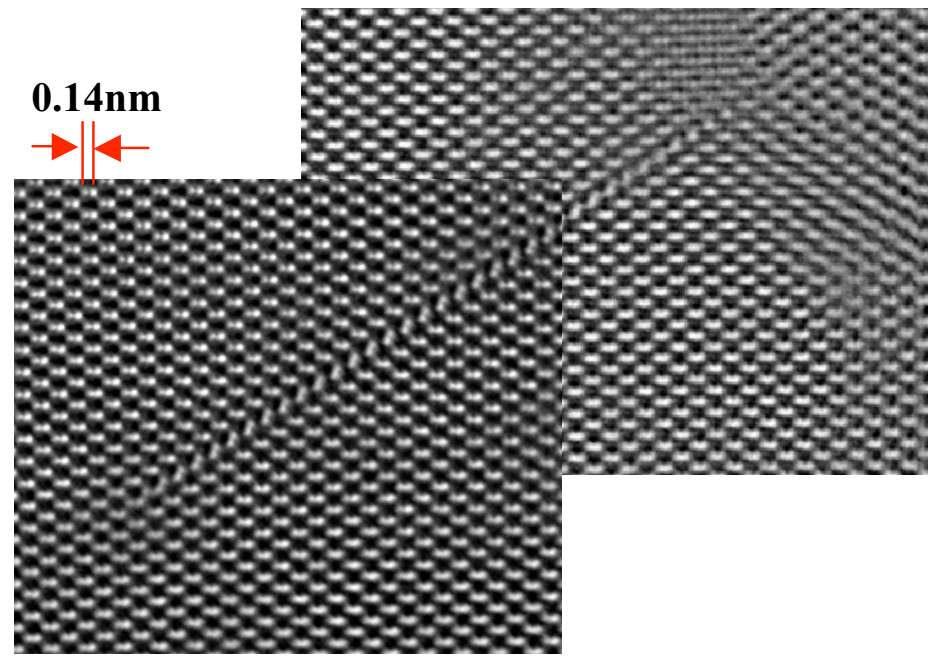

FIG. 1. Phase image of the reconstructed electron exit wave with two $30^{\circ}$ partial dislocations at the ends of a stacking fault in GaAs: Be (Projection orientation [011]).

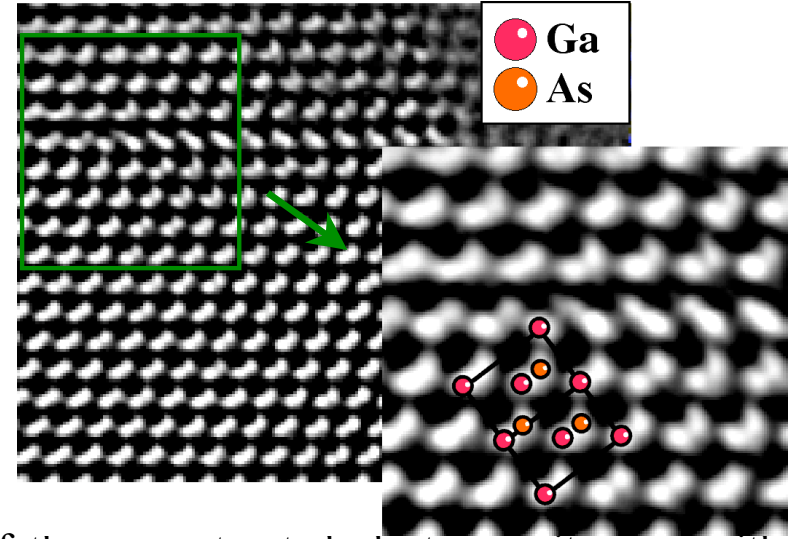

FIG. 2. Phase image of the reconstructed electron exit wave with stacking faults and partial dislocation in GaAs: Be (Projection orientation [011]). The inset shows enlarged image of the partial dislocation at the end of an intrinsic stacking fault.

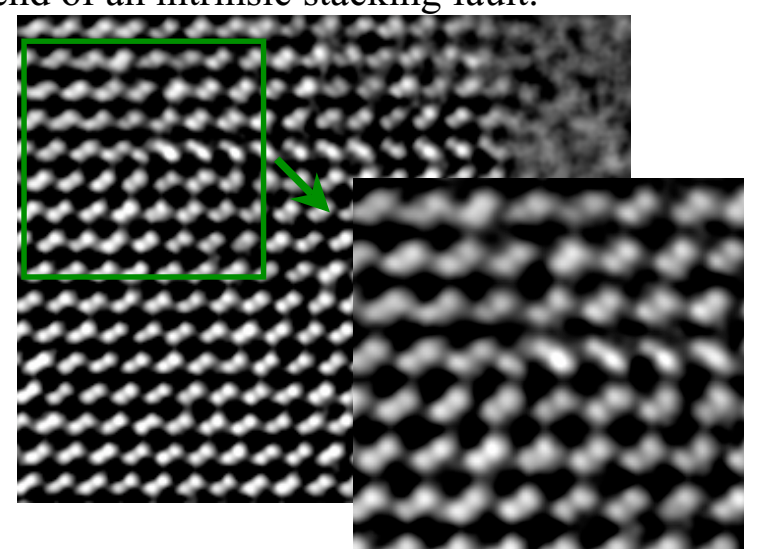

FIG. 3. Phase image of the reconstructed electron exit wave with stacking faults and partial dislocation in GaAs: Be (Projection orientation [011]) by aberration correction with software. 